\title{
Le sexe et les SOX
}

\section{Philippe Jay Catherine Gozé Stephan Soullier Marion Desclozeaux Francis Poulat Vincent Laudet Philippe Berta}

\section{ADRESSES}

P. Jay: boursier MRE. C. Gozé: docteur en pharmacie et en biochimie. S. Soullier: étudiant en thèse de biochimie. M. Desclozeaux : boursière MRE. F. Poulat: stagiaire post-doctoral. $\mathrm{P}$ Berta : directeur de recherche à l'Inserm. Centre de recherche de biochimie macromoléculaire, Cnrs UPR9008/Inserm U. 249, route de Mende, BP 5051, 34033 Montpellier Cedex, France. V. Laudet: chargé de recherche au Cnrs. Cunrs URAl160, oncologie moléculaire, Institut Pasteur de Lille, 1, rue Calmette, 59019 Lille Cedex, France.
La découverte du facteur de détermination testiculaire SRY a permis la caractérisation d'une nouvelle famille de facteurs transcriptionnels, les protéines SOX. Ces facteurs, extrêmement conservés au cours de l'évolution, furent en effet identifiés à l'origine comme les produits des gènes présentant une boîte de liaison à l'ADN de type HMG, très similaire à celle de $\mathrm{SRY}$. Les nombreux gènes $S O X$ désormais décrits, apparaissent exprimés dans les tissus embryonnaires et sont très probablement impliqués dans la régulation d'étapes précoces du développement. Si des éléments communs caractérisent leur mode d'action (liaison à des séquences $\mathrm{ADN}$ riches en A-T, courbure de l'ADN, liaison au petit sillon de l'ADN), peu de choses sont encore connues sur la nature des gènes cibles réglés par les $\mathrm{SOX}$, et au-delà sur leur fonction précise. Néanmoins, certains gènes $S O X$ ont déjà pu être impliqués dans diverses anomalies du développement telles la réversion du sexe ou la dysplasie campomélique. a famille des gènes $S O X$ a été mise à jour lors du clonage, en 1990, du gène $S R Y$ codant pour un facteur de détermination testiculaire humain $\left(\mathrm{m} / \mathrm{s} n^{\circ} 11\right.$, vol. 9, p. 1247) [1-4]. En effet, si le gène $S R Y$ code bien pour une protéine présentant un motif protéique de liaison à l'ADN de type domaine HMG (high mobility group), la protéine SRY a, en outre, permis de définir un nouveau sous-groupe au sein de la famille des protéines à motif HMG. Rappelons que les protéines à domaine $\mathrm{HMG}$ regroupent une famille de protéines nucléaires non histone dont le mode d'interaction avec l'ADN présente des caractéristiques communes (interaction avec le petit sillon de l'ADN, courbure de l'ADN, liaison à de l'ADN cruci- forme). Le nombre de domaines HMG présents dans la protéine, la capacité de ces protéines de reconnaître ou non des séquences spécifiques, permettent aujourd'hui d'en réaliser une classification. Si toutes les protéines à domaine HMG semblent jouer un rôle architectural au cœur de la chromatine, certaines à domaine unique apparaissent clairement participer à la réalisation de complexes multiprotéiques réglant la transcription de gènes cibles. Ainsi en est-il des protéines SOX (Sry box containing), qualifiées ainsi en raison d'une conservation en acides aminés supérieure à $60 \%$ entre leur motif HMG et celui de la protéine SRY. Comme nous le verrons, ces critères de séquence sont aujourd'hui complétés par des critères fonctionnels 


\section{RÉFÉRENCES}

1. Sinclair AH, Berta P, Palmer MS, Hawkins JR, Griffiths BL, Smith MJ, Foster JW, Frischauf AM, Lovell-Badge R, Goodfellow PN. A gene from the human sex-determining region encodes a protein with homology to a conserved DNA-binding motif. Nature $1990 ; 346$ : 240-4.

2. Gubbay J, Collignon J, Koopman P, Capel B, Economou A, Münsterberg A, Vivian N, Goodfellow P, Lovell-Badge R. A gene mapping to the sex-determining region of the mouse $Y$ chromosome is a member of a novel family of embryonically expressed genes. Nature $1990 ; 346$ : 245-50.

3. Weissenbach J, Petit C. Chromosome Y et détermination du sexe. médecine/sciences $1990 ; 6: 785-90$

4. Barbaux S, Vilain E, McElreavey $\mathrm{K}$, Fellous M. Le point sur le déterminisme du sexe chez les mammifères. médecine/sciences $1995 ; 11: 529-36$.

5. Palmer MS, Sinclair AH, Berta P, Ellis NA, Goodfellow PN, Abbas NE, Fellous M. Genetic evidence that ZFY is not the testisdetermining factor. Nature 1989; 342: 937-9.

6. Berta P, Hawkins JR, Sinclair AH, Taylor A, Griffiths BL, Goodfellow PN, Fellous M. Genetic evidence equating SRY and the testis determining factor. Nature $1990 ; 348$ : 448-50.

7. Koopman P, Gubbay J, Vivian N, Goodfellow PN, Lovell-Badge $R$. Male development of chromosomally female mice transgenic for Sry. Nature 1991; 351 : 117-21.

8. Behlke MA, Bogan JS, Beer-Romero $P$ Page DC. Evidence that the SRY protein is encoded by a single exon on the human Y chromosome. Genomics 1993; 17: 736-9.

9. Clepet C, Schafer AJ, Sinclair AH, Palmer MS, Lovell-Badge R, Goodfellow PN. The human SRY transcript. Hum Mol Genet 1993; 2: 2007-12.

10. Su H, Lau YFC. Identification of the transcriptional unit structural organization, and promotor sequence of the human sexdetermining region $\mathrm{Y}(S R Y)$ gene, using a reverse genetic approach. Am J Hum Genet $1993 ; 52: 24-38$

11. Koopman P, Münsterberg A, Capel B, Vivian N, Lovell-Badge R. Expression of a candidate sex-determining gene during mouse testis differentiation. Nature 1990 ; 348: $450-2$

12. Harry JL, Koopman P, Brennan FE, Marshall Graves JA, Renfree MB. Widespread expression of the testis-determining gene $S R Y$ in a marsupial. Nature Genet $1995^{\circ}$ 11: 347-9.

13. Poulat F, Girard F, Chevron MP, Gozé C, Rebillard X, Calas B, Lamb N, Berta P. Nuclear localization of the testis determining gene product SRY. I Cell Biol 1995 $128: 737-48$ qui participent à la définition de cette nouvelle sous-famille. Le gène $S R Y$ est donc un élément particulier de cette sous-famille qui, du fait de sa localisation sur le chromosome $\mathrm{Y}$, a évolué vers une fonction particulière, la détermination du sexe chez les mammifères.

Au cours de cet article de synthèse, il ne sera déjà plus possible d'être exhaustif sur l'ensemble des gènes $S O X$ présents dans les banques de données dont les séquences sont, en outre, le plus souvent partielles. Nous nous limiterons, en fait, à énoncer les propriétés connues du $S O X$ le plus étudié, le gène $S R Y$ et la protéine correspondante, et à récapituler les récentes avancées effectuées dans la caractérisation de certains gènes SOX.

\section{La protéine SRY humaine}

Le clonage positionnel en 1990 du gène $S R Y$ a fait suite à de longues années de recherche et de nombreuses fausses pistes [1]. Ce clonage a été permis par les progrès de la cartographie, dans le cas présent celle du chromosome Y, qui, fournissant de nouvelles sondes, a permis d'affiner l'analyse moléculaire d'une forme particulière d'ambiguïté sexuelle ou réversion sexuelle $46, \mathrm{XX}$ [5]. Le gène $S R Y$ est un petit gène monoexonique codant pour une protéine de 204 acides aminés. Après l'analyse de banques protéiques, SRY se révéla présenter une faible analogie structurale avec différentes protéines appartenant toutes à la famille des protéines de haute mobilité [1]. L'homologie est restreinte au domaine HMG, soit environ 80 acides aminés occupant une position relativement centrale dans SRY (figure 1). La présence de la boîte $\mathrm{HMG}$ a permis de postuler très tôt une fonction de liaison à l'ADN pour SRY. Par la suite, les nombreuses expériences génétiques et moléculaires établirent l'identité de SRY avec le facteur de détermination testiculaire TDF (testis determining factor) [3, 6, 7].

\section{Profil d'expression du gène $S R Y$ humain}

Si le gène $S R Y$ est monoexonique [8], les sites de début de sa transcription décrits dans la littérature appa- raissent multiples [8-10]. Des études ont montré que, chez l'embryon de souris, son expression est restreinte au moment même de la mise en place des testicules [11], ainsi que chez les marsupiaux [12]; en revanche, les données sur l'expression du gène $S R Y$ dans l'espèce humaine restent peu nombreuses. Les seules données embryonnaires résultent de l'analyse par immunofluorescence de la présence de la protéine SRY dans une section d'embryon d'environ six semaines au niveau des structures gonadiques [13]. Chez le foetus et l'adulte, des études par RT-PCR révèlent la présence de transcrits, avec des disparités quantitatives probables dans de nombreux tissus mâles. De récents travaux effectués en Allemagne confirment la présence de transcrits $S R Y$ dans le système nerveux [14]. Si elle est confirmée, la fonction d'une telle expression extragonadique reste énigmatique.

Modes d'action de la protéine SRY: premières données

A l'appui des prédictions résultant de l'analyse de la séquence de la protéine SRY, des essais in vitro démontrèrent sa liaison spécifique à des séquences de type AACAAAG également reconnues par d'autres membres de la famille des $\mathrm{HMG}$ comme TCF-1 (T cell-specific transcription factor) ou LEF-1 (lymphoid enhancer factor) [15]. Plus tard, par sélection directe d'oligonucléotides, une séquence consensus optimale (A/T)AACAAT fut définie, SRY présentant pour cette dernière une affinité environ cing fois supérieure à celle observée pour le site TCF1/LEF-1 [16]. Cependant, à ce jour, aucune confirmation in vivo par détermination de gènes cibles pour SRY n'est venue appuyer ces premiers résultats. Une approche à l'aide de peptides synthétiques confirme que, si la boîte $\mathrm{HMG}$ de SRY contrôle bien cette activité de liaison à l'ADN [17], elle pilote également la localisation nucléaire de la protéine [13]. Pour SRY comme pour LEF-1, la liaison à l'ADN implique le petit sillon et s'accompagne d'une forte torsion de ce dernier [18]. Cette observation pourrait conforter l'hypothèse que SRY, par sa 


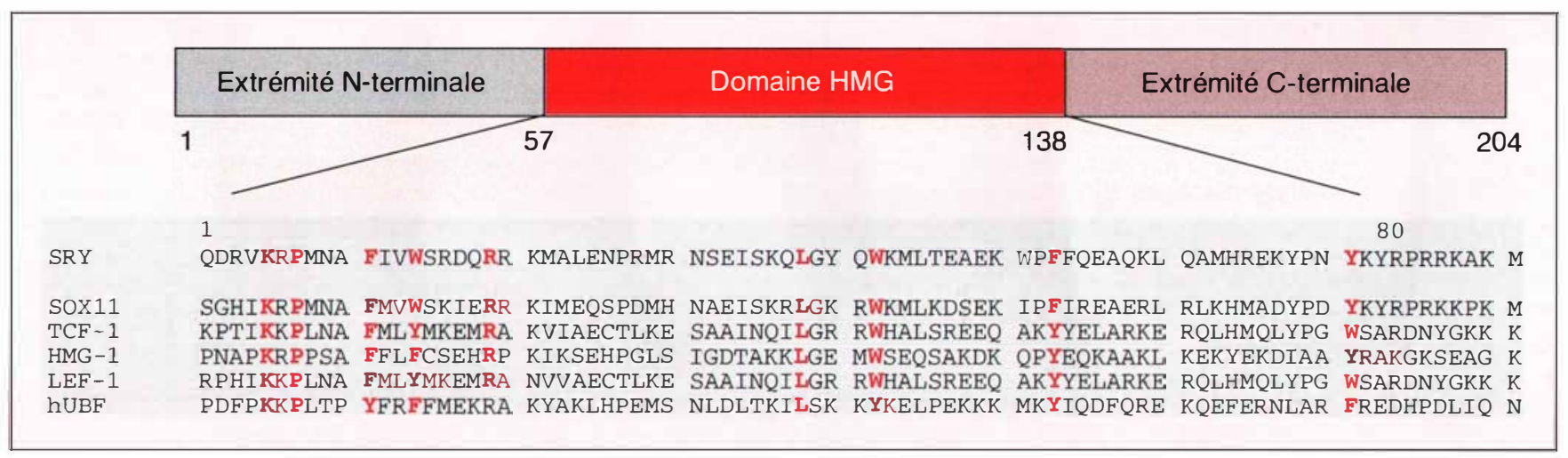

Figure 1. La protéine SRY contient en son centre un domaine de liaison à I'ADN de type boîte HMG. Les acides aminés conservés sont indiqués en rouge. Code à une lettre des acides aminés : $A$ : Ala; C: Cys ; D: Asp; $E$ : Glu ; $F$ : Phe ; G: Gly; H: His; I: Ile; K:Lys;L:Leu; M: Met; N:Asn; P: Pro; S: Ser; T: Thr;V: Val; W: Trp; Y : Tyr.

liaison, rapprocherait des séquences d'ADN et des facteurs protéiques associés, qui étaient jusque-là séparés par une distance linéaire importante. Cette action permettrait alors la réalisation d'un complexe transcriptionnel multiprotéique. Enfin, la protéine SRY apparaît capable de lier in vitro l'ADN simple brin, indépendamment de sa séquence pourvu que ce dernier soit sous forme structurée comme dans l'ADN cruciforme [19]. Si le domaine HMG de SRY est responsable de sa liaison spécifique à l'ADN, de la courbure de l'ADN et de sa localisation nucléaire, les acides aminés se trouvant en dehors de ce domaine n'ont pas trouvé, à ce jour, de rôle fonctionnel, tout du moins chez l'homme. Une capacité transactivatrice potentielle a été détectée pour ces derniers chez la souris [20], mais sa réalité physiologique reste à démontrer.

Par des méthodes in vitro, des sites de liaison pour SRY ont été décrits dans de nombreux promoteurs de gènes dont certains interviennent dans la cascade de détermination/différenciation du sexe chez l'homme. Ainsi en est-il des promoteurs des gènes codant pour l'aromatase P450 ou l'hormone antimüllérienne (AMH). De plus, la coïncidence des profils d'expression de $S R Y$ et du gène de l'AMH conforte aujourd'hui l'hypothèse d'un contrôle de l'expression de ce dernier par la protéine SRY [21]. Cet effet, probablement indirect, semble nécessiter en outre un cofacteur de nature inconnue [22]. Enfin, une séquence consensus pour SRY est également présente dans le promoteur du gène fra-1 (Fos-relaated antigen-1), gène codant pour un composant du facteur de transcription AP-l qui se trouve exprimé, au cours du développement, dans de nombreux processus, dont la spermatogenèse. Des expériences de transactivation ont confirmé la capacité d'activation par la protéine SRY du gène fra-1 [23]. Là encore, la démonstration de cette activité in vivo reste à fournir.

\section{Conservation de la protéine SRY chez les mammifères}

Si le rôle de la protéine SRY comme facteur de détermination testiculaire porté par le chromosome $\mathrm{Y}$ est aujourd'hui admis, la conservation et l'origine même du gène $S R Y$ restent sources de spéculation. On a retrouvé à ce jour $S R Y$ porté par le chromosome Y de tous les mammifères étudiés. Cependant, chez certains rongeurs, SRY n'a pas été retrouvé, faute de chromosome $Y$ [24]. La conservation des SRY des différents mammifères se limite essentiellement au domaine HMG (figure 2). La grande divergence des séquences protéiques situées de part et d'autre de ce domaine est très surprenante, en particulier pour un gène localisé dans une région chromosomique où les polymorphismes sont rares [25, 26]. Cette divergence forme la base de nombreuses hypothèses [27, 28]. Des îlots de conservation dans les domaines carboxy- et aminoterminaux semblent cependant persister, et tout rôle fonction- nel n'est donc pas à exclure. Comme nous le verrons, la réduction, voire l'absence, de conservation des domaines hors boîte HMG des différents SRY leur est spécifique, et est inhabituelle dans la famille SOX.

\section{Les protéines SOX}

Les premiers gènes $S O X$ définis par la présence dans leurs produits d'une boîte HMG proche de SRY (au moins $60 \%$ de conservation des acides aminés avec le domaine HMG de SRY) ont été tout d'abord partiellement décrits chez la souris [2] ; ce résultat a été obtenu par clonage, à partir d'une banque d'expression réalisée à l'aide d'embryons de souris de 8,5 jours post-coïtum, en utilisant comme sonde la séquence codant pour la boîte HMG de SRY. Depuis, le répertoire des gènes $S O X$ n'a cessé de croître (figure 3). Les informations obtenues pour la majorité d'entre eux se limitent à la séquence partielle codant pour leur boîte HMG, séquence obtenue par utilisation de la PCR dégénérée fondée sur le haut degré de conservation de ces boîtes de type SRY (pour une revue, voir [29]). Cependant, bien que les gènes SOX décrits avec plus de détails soient rares, ces études suggèrent, comme pour SRY, un rôle clé pour leurs produits comme facteurs de transcription qui interviendraient dans la régulation de grandes voies de développement. Ainsi, par exemple, pourraient-ils contribuer à la mise en place du système nerveux [30, 31], du cour [32], à la différen- 


\section{RÉFÉRENCES}

14. Lahr G, Maxson SC, Mayer A, Just W Pilgrim C, Reisert I. Transcription of the $Y$ chromosomal gene, $S r y$, in adult mouse brain. Mol Brain Res 1995; 33: 179-82.

15. Harley VR, Jackson DI, Hextall PJ, Hawkins JR, Berkovitz GD, Sockanathan S, Lovell-Badge R, Goodfellow PN. DNA binding activity of recombinant SRY from nor$\mathrm{mal}$ males and XY females. Science 1992; 255: 453-6.

16. Harley VR, Lovell-Badge R, Goodfellow PN. Definition of a consensus DNA binding site for SRY. Nucleic Acids Res 1994; 22: 1500-1.

17. Poulat F, Guichard G, Gozé C, Heitz F, Calas B, Berta P. Synthesis of a large peptide mimicking the DNA binding properties of the sex determining protein, SRY. FEBS Lett 1992 ; 309 : 385-8.

18. Giese K, Cox J, Grosscheld R. The HMG domain of lymphoid enhancer factor 1 binds DNA and facilitates assembly of functional nucleoprotein structures. Cell 1992; 69: 185-95.

19. Pontiggia A, Rimini R, Harley VR, Goodfellow PN, Lovell-Badge R, Bianchi ME. Sexreversing mutations affect the architecture of SRY-DNA complexes. EMBO I 1994; 13: 6115-24.

20. Dubin RA, Ostrer $H$. Sry is a transcriptional activator. Mol Endocrinol 1994; 8: 1182-92

21. Haqq CM, King CY, Ukiyama E, Falsafi S, Haqq TN, Donahoe PK, Weiss MA. Molecular basis of mammalian sexual determination : activation of Müllerian inhibiting substance gene expression by SRY. Science 1994 ; 266: 1494500 .

22. Shen WH, Moore CCD, Ikeda Y, Parker $\mathrm{KL}$, Ingraham HA. Nuclear receptor steroidogenic factor 1 regulates the Müllerian inhibiting substance gene: a link to the sex determination cascade. Cell 1994; 77 : 651-61.

23. Cohen DR, Sinclair AH, McGovern JD. Sry protein enhances transcription of Fosrelated antigen-1 promoter constructs. Proc Natl Acad Sci USA 1994; 91 : 4372-6.

24. Just W, Rau W, Vogel W, Akheverdian M, Fredga K, Marshall Graves JA, Lyapunova $\mathrm{E}$. Absence of Sry in species of the vole ellobius. Nature Genet 1995; 11 : 117-8.

25. Malaspina $P$, Persichetti F, Novelletto A, Iodice C, Terrenato L, Wolfe J, Ferraro M, Prantera. The Y chromosome shows a low level of DNA polymorphism. Ann Hum Genet 1990; 54 : 297-305.

26. Ellis NA, Taylor A, Bengtsson BO, Kidd J, Rogers J, Goodfellow P. Population structure of the human pseudoautosomal boun-

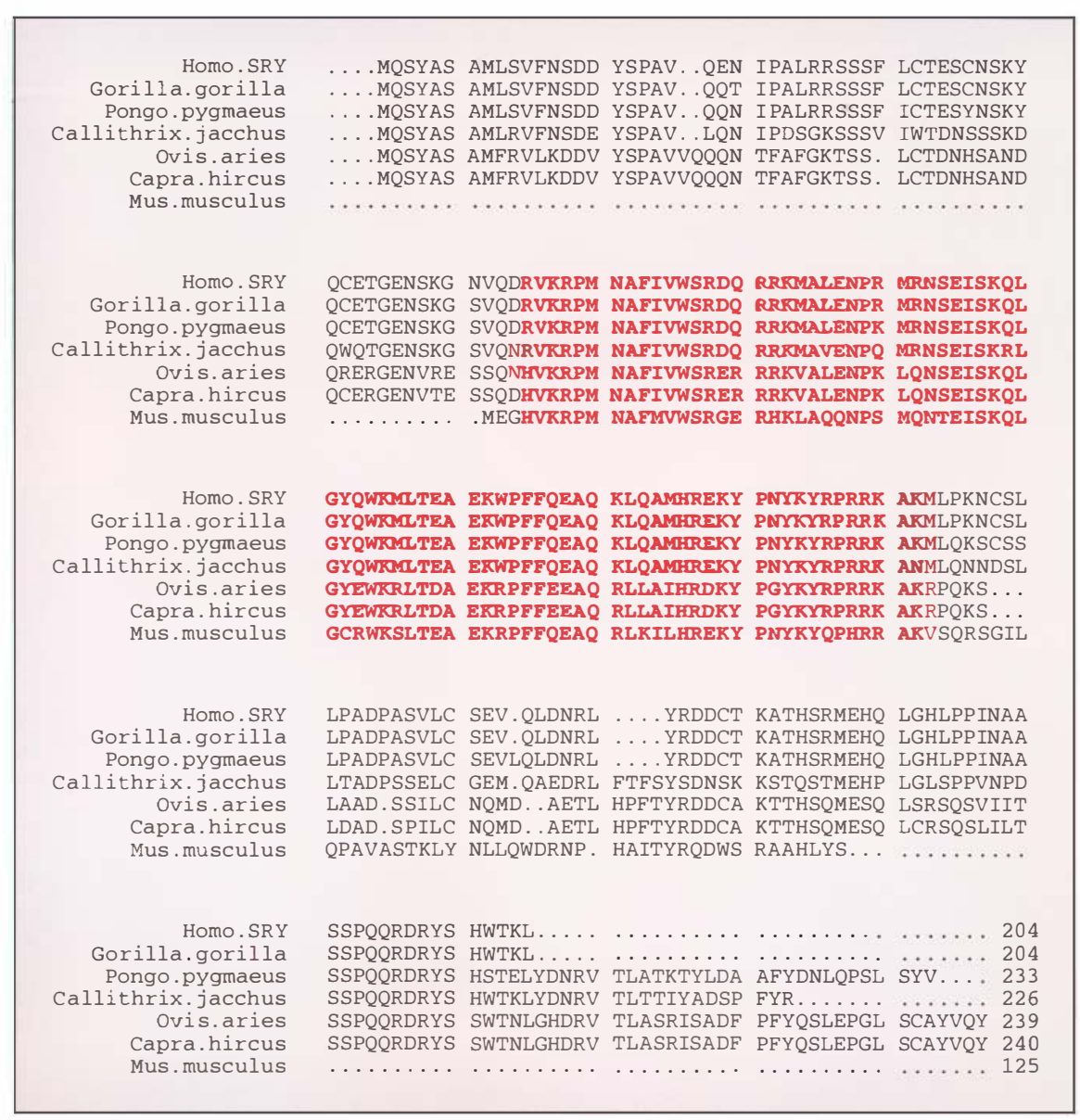

Figure 2. Conservation de séquences entre différentes protéines SRY chez différents mammifères. Si le motif HMG inscrit en rouge apparaît très conservé, des éléments de conservation sont présents hors boîte lorsque l'on exclut les séquences SRY de rongeurs et de mammifères. Code à une lettre des acides aminés: A : Ala ; C : Cys ; D: Asp; E: Glu; F: Phe; G: Gly; $H$ : His ; I: lle ; K: Lys ; L: Leu; M: Met ; N: Asn; P : Pro; S : Ser; T: Thr; V: Val; W:Trp; Y: Tyr.

ciation des lymphocytes $\mathrm{T}$ et $\mathrm{B}$ [33], à la formation des os [34] ou encore au déroulement de la spermatogenèse $[35,36]$.

Premiers éléments de conservation et de classification

Les gènes $S O X$ apparaissent extrêmement conservés au cours de l'évolution. Il serait fastidieux d'établir la liste des multiples locus détectés et de la diversité des espèces concernées. Citons pour mémoire le nématode, la drosophile, les poissons, les reptiles, les oiseaux et les mammifères (figure 3). La multiplication des séquences aujourd'hui répertoriées permet d'envisager la mise en place d'un arbre phylogénétique à part entière pour ce sous-groupe de pro- téines à domaine HMG (Soullier et $a l$, manuscrit en préparation). Un arbre bâti dès 1993 fait déjà apparaître des regroupements possibles à l'intérieur même de la famille des SOX [37]. Un arbre partiel établi à l'aide des séquences de boîtes HMG de différents SOX le confirme (figure 4). La structure de cet arbre, le nombre important de gènes SOX ainsi que la structure monoexonique de la grande majorité d'entre eux ont permis de proposer l'hypothèse d'une duplication spécifique de certains gènes SOX dans certains groupes zoologiques. Par exemple, la séquence du gène $S_{0 X 3} 3$ (gène localisé sur le chromosome $\mathrm{X}$ humain et candidat pour une forme particulière de retard mental lié à l'X) révèle une très forte similitude avec $S R Y$ au 


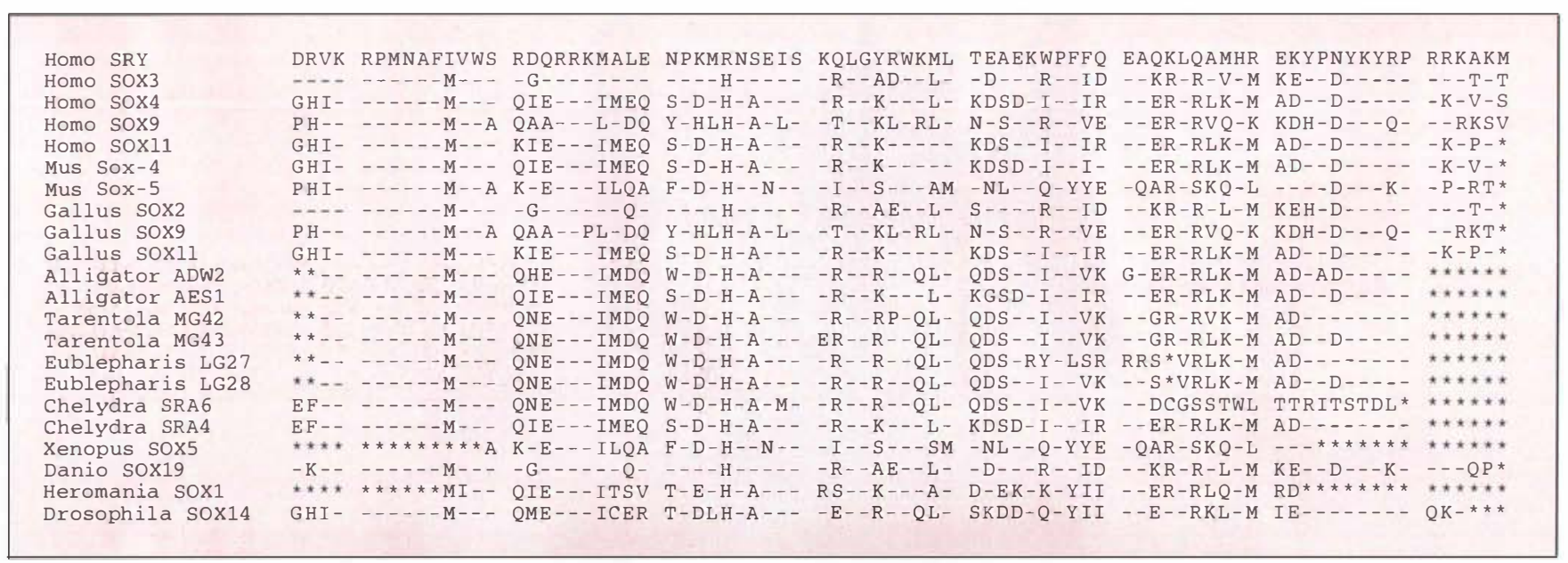

Figure 3. Conservation des boîtes SOX dans de nombreuses espèces. * : Acide aminé non déterminé; - : identité définie en prenant la séquence du SRY humain comme référence.

niveau de la séquence codant pour le domaine HMG [38]. Ces deux gènes localisés sur les gonosomes pourraient être issus d'un ancêtre commun. De même, les gènes SOX11 et SOX4 sont très similaires, et cette similitude s'étend bien au-delà du seul motif de liaison à l'ADN [31]. Les gènes $S O X 9$, SOX18 et tout récemment $S O X 17$ (non incorporé dans cette étude) se caractérisent par la présence d'une séquence intronique au sein de la séquence codant pour le domaine HMG. L'existence d'un ancêtre commun à ces trois gènes est très probable. Enfin, pour les quelques exemples pour lesquels ce travail est possible, l'alignement d'un même gène $S O X$ de différentes espèces démontre une importante conservation du gène tout au long de sa séquence. C'est le cas, par exemple, du gène SOXI1 (figure 5). Comme déjà mentionné, seul le gène $S R Y$ échappe à cette règle. Enfin, toutes les protéines SOX analysées apparaissent capables de reconnaître une séquence d'ADN similaire à celle reconnue par SRY, leur liaison conduisant également à une courbure comparable de l'ADN cible. Des expériences récentes de notre groupe n'excluent cependant pas la possibilité d'une affinité différentielle des boîtes SOX. La liaison de ces boîtes peut, en particulier, être sensible à la nature des séquences adjacentes au site ADN accepté à ce jour comme consensus; la diversité de leur fonction pourra également être recherchée à travers la nature des séquences des protéines SOX localisées en dehors de leur domaine de liaison à l'ADN; ou encore dans le type de combinatoire de facteurs transcriptionnels dans laquelle une protéine SOX particulière se trouve impliquée; ou, enfin, dans leur profil d'expression spatio-temporelle.

\section{Modes d'action et fonctions des gènes $\mathrm{SOX}$}

Ce point sera abordé à travers deux des rares exemples qui commencent à être bien documentés, les gènes SOX2 et SOX9.

\section{- $S O X 2$}

Parmi les premiers gènes $S O X$ décrits, SOX1, SOX2 et SOX3 sont exprimés dans l'embryon de souris essentiellement dans le système nerveux et, plus particulièrement pour SOX1 et $S O X 2$, au niveau de l'oil [30]. Un ADNc codant pour une protéine identique à SOX-2 fut ensuite cloné chez l'homme et localisé sur le chromosome 3 en q26.3-27 [39]. Deux fonctions fort différentes viennent d'être attribuées au gène $S O X 2$, l'une utilisant l'homologue de poulet, et l'autre l'homologue murin. La première est issue des études du réglage de l'expression des gènes codant pour les protéines du cristallin; elles viennent de permettre, pour la première fois, d'attribuer une cible génétique à un membre de la famille des SOX [40]. Le réglage spécifique, dans le cristallin, du gène codant pour la protéine $\delta 1$ du cristallin est sous le contrôle d'une séquence activatrice de 30 paires de bases ou fragment DC5. Son étude par mutagenèse a montré que cette séquence DC5 serait formée, entre autres, de deux éléments contigus interdépendants exerçant un contrôle positif. L'élément situé le plus en 5', ou élément DC13, est capable de lier un groupe de facteurs nucléaires notés $\delta E F 2 a-d$. C'est dans le cristallin due le facteur $\delta E F 2 a$ est de loin le plus abondant, mais il est également synthétisé à un faible niveau dans le cerveau. L'utilisation de la séquence DC13 sous forme multimère pour cribler une banque d'expression préparée à partir des ARNm de cristallin d'un embryon de poulet de 13 jours a permis le clonage de cSOX-2 [41]. Différents arguments indirects permettent d'identifier cSOX-2 et $\delta E F 2 a$ (comparaison de la protéine $\delta E F 2 a$ semi-purifiée et de la protéine cSOX-2, comparaison des profils d'expression de leurs gènes). Enfin, la capacité d'activation de la séquence enhancer du gène de la protéine $\delta 1$ par cSOX-2 fut testée par une approche, désormais classique, de mesure d'activité d'un gène rapporteur, après transfection dans différentes cellules de diverses constructions associant séquence DC13 sauvage ou mutée et gène cSOX-2. Par ailleurs, ces résultats qui impliquent une protéine SOX dans la 


\section{RÉFÉRENCES}

27. Whitfield LS, Lovell-Badge R, Goodfellow PN. Rapid sequence evolution of the mammalian sex-determining gene $S R Y$. Nature $1993 ; 364: 713-5$.

28. Hurst LD. Embryonic growth and the evolution of the mammalian $Y$ chromosome. II. Suppression of selfish Y-linked growth factors may explain escape from $\mathrm{X}$ inactivation and rapid evolution of Sry. Heredity 1994; 73: 233-43.

29. Gozé C. Les gènes $S O X$ : clonages, caractérisations et conservation dans le règne animal. Thèse de doctorat, Montpellier I, France, septembre 1995.

30. Sockanathan S, Cohen-Tannoudji M, Collignon I, Lovell-Badge R. Characterization of SOX-1, -2 and -3 : DNA binding proteins expressed in the developing central nervous system of the mouse. Genet Res $1993 ; 61: 149$.

31. Jay P, Gozé C, Marsollier C, Taviaux S, Hardelin JP, Koopman P, Berta P. The human SOX11 gene: cloning, chromosomal assignment and tissue expression. Genomics 1995 ; $29: 541-5$.

32. Schilham MW, Oosterwegel MA, Moerer $P$, Ya J, de Boer PAJ, van de Wetering $M$, Verbeek S, Lamers WH, Kruisbeek AM, Cumano A, Clevers $H$. Defects in cardiac outflow tract formation and pro-B-lymphocyte expansion in mice lacking SOX-4. Nature 1996, 380: 711-4.

33. Van de Wetering $M$, Oosterwegel $M$, van Norren K, Clevers H. SOX-4, an Sry-like HMG box protein, is a transcriptional activator in lymphocytes. $E M B O=1993 ; 12$ : 3847-54.

34. Foster JW, Dominguez-Steglich MA, Guioli S, Kwok C, Weller PA, Stevanovic M, Weissenbach J, Mansour S, Young ID, Goodfellow PN, Brook JD, Schafer AI. Campomelic dysplasia and autosomal sex reversal caused by mutations in an SRY-related gene. Nature 1994 ; 372 : 525-30.

35. Denny P, Swift S, Connor F, Ashworth A. An $S R Y$-related gene expressed during spermatogenesis in the mouse encodes a sequence-specific DNA-binding protein. EMBO J 1992; 11 : 3705-12.

36. Kanai Y, Kanai-Azuma M, Noce T, Saido TC, Shiroishi T, Hayashi Y, Yazaki K Identification of two SOX 17 messenger RNA isoforms, with and without the high mobility group box region, and their differential expression in mouse spermatogenesis. $J$ Cell Biol 1996; 133: 667-81.

37. Laudet V, Stehelin D, Clevers H. Ancestry and diversity of the HMG box superfa-

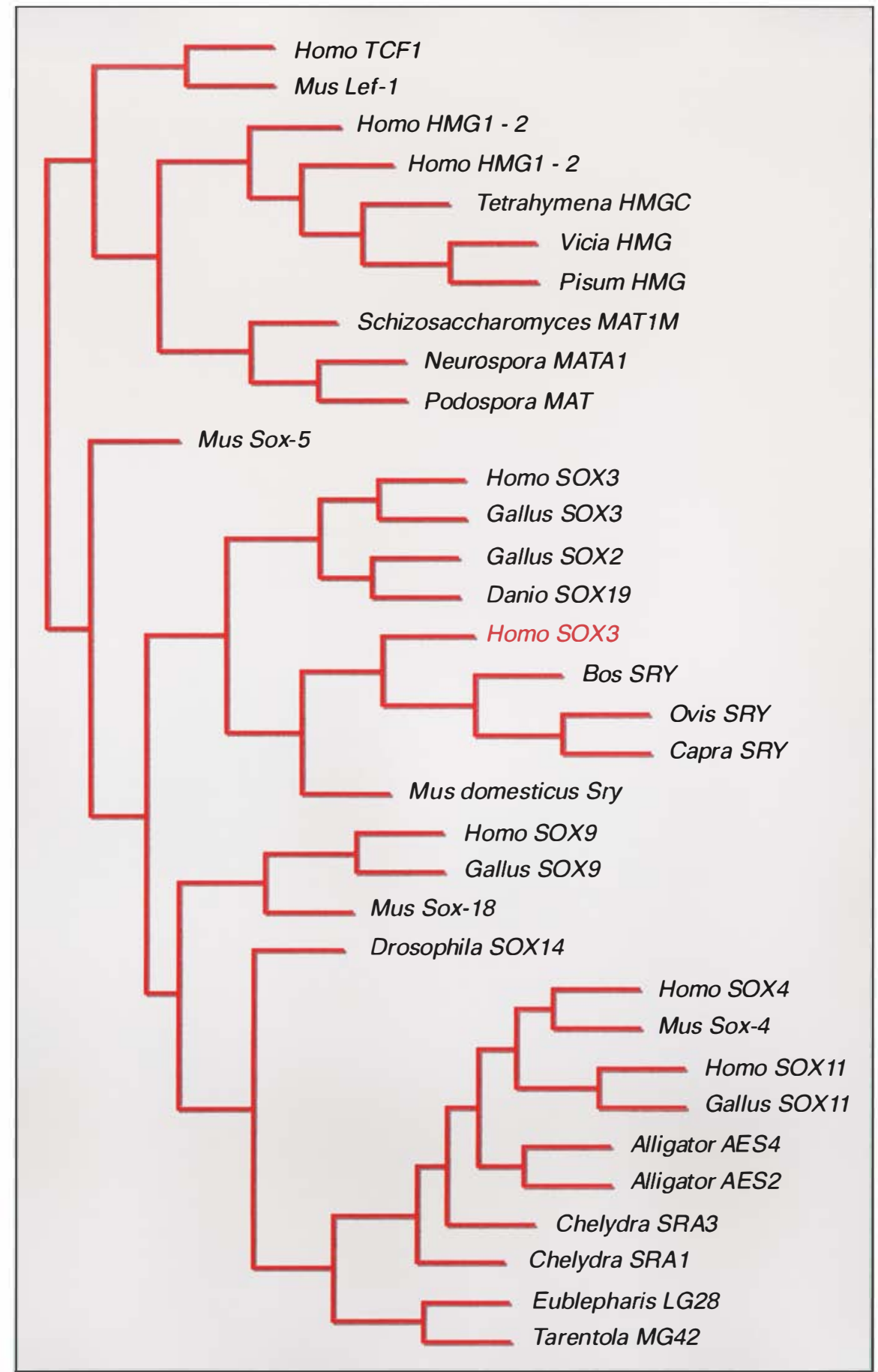

Figure 4. Arbre phylogénétique partiel des domaines HMG de quelques gènes SOX. Cet arbre a été réalisé par la méthode du neighbourg joining du logiciel MUST (méthode bootstrap) [47, 48].

régulation de gènes exprimés dans le cristallin ont pu être étendus au modèle souris où SOX-1 ou SOX-2, ou encore les deux, semblent impliqués [40]. Il reste à noter que dans ce cas encore, l'activation du enhancer par cSOX-2 n'a pu s'effectuer sans la présence de partenaires, d'où la nécessité d'utiliser un modèle cellulaire spécifique dérivé du cristallin. Comme LEF-1 [17], SOX2 et, audelà, très probablement l'ensemble des protéines SOX, agirait en facilitant la formation d'un complexe 


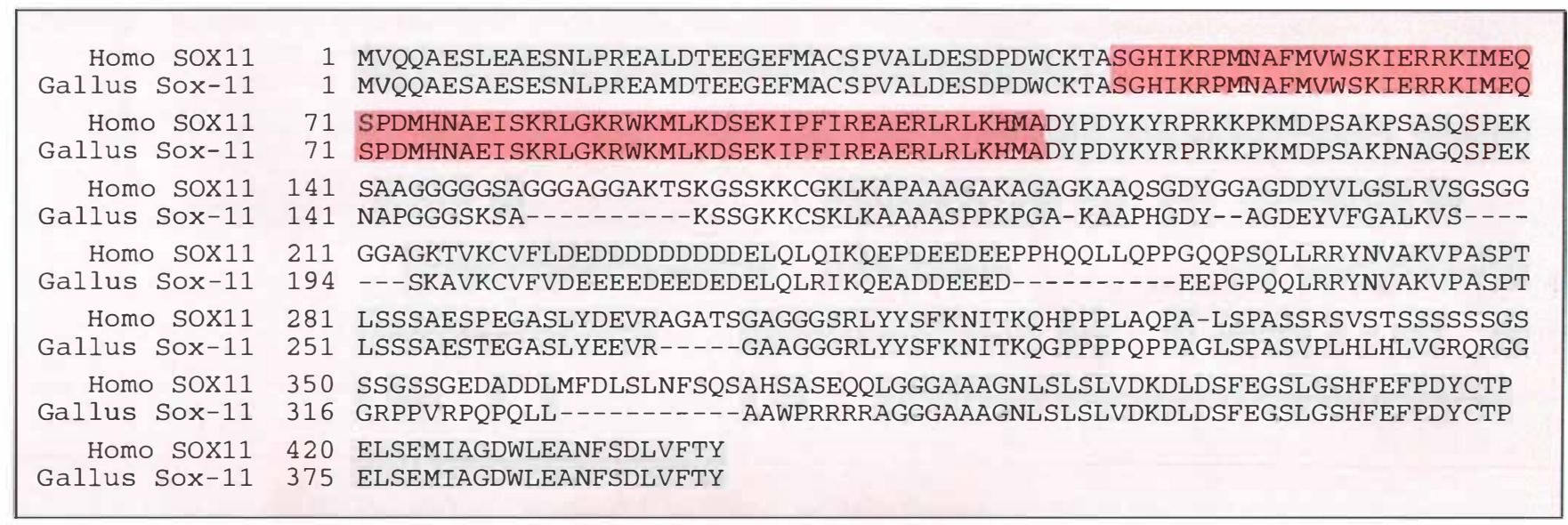

Figure 5. La majorité des gènes SOX apparaissent conservés tout au long de leur séquence (boîte et hors boîte); un exemple: le gène SOX11. Gallus: poulet. La boîte HMG apparaît en rose.

multiprotéique dont certains éléments peuvent être, eux aussi, spécifiques de types cellulaires donnés. Parallèlement à ce travail, une autre équipe vient d'impliquer le gène SOX2 de souris plus précocement au cours de l'embryogenèse dans une activité régulatrice bien différente [41], par la recherche d'éléments contrôlant la synthèse du facteur de croissance des fibroblastes FGF-4, un facteur essentiel pour la survie de l'embryon post-implantatoire et à des stades plus âgés. Ce facteur est synthétisé dans la masse cellulaire interne du blastocyste et, plus tard, dans différents tissus embryonnaires alors qu'on ne le trouve pas chez l'adulte. Les cellules souches dérivées de carcinome embryonnaire (EC) constituent un bon modèle pour l'étude de la régulation du gène $F G F$ 4. Cette expression est gouvernée par une combinatoire de facteurs se liant à différents éléments en cis dans les séquences enhancer, parmi lesquels Oct-1, Oct-3 et le facteur Fx. Après clonage du gène $S O X 2$ à partir de cellules EC, différents arguments permirent d'établir l'identité de SOX2 et du facteur Fx. Là encore, la transactivation du enhancer de FGF-4 n'a pu être obtenue que dans un contexte cellulaire particulier (en l'occurrence des cellules indifférenciées), et des expériences de co-transfection ont mis en lumière que seule la réalisation d'un complexe SOX2/Oct-3 peut activer la transcription du gène FGF-4.

$\mathrm{Si}$, pour SOX2, comme pour d'autres protéines SOX, ou plus largement pour les protéines à domaine $\mathrm{HMG}$, la réalisation d'un complexe multiprotéique semble la règle, la possibilité pour un même facteur SOX d'intervenir dans différentes cellules et à différentes périodes du développement embryonnaire est mise en lumière par ces deux études. Aucun profil d'expression spatio-temporelle de SOX2 n'est encore venu corroborer ces résultats.

\section{- $S O X 9$}

La dysplasie campomélique ou campomélisme est un syndrome malformatif associant anomalies des os et des cartilages avec fréquemment réversion sexuelle de type fille 46,XY. L'analyse de translocations chromosomiques associées parfois à ce syndrome a permis, dans un premier temps, la définition d'un locus autosomique pour une réversion sexuelle ou locus SRA1 (autosomal sex reversal locus) associé au locus dysplasie campomélique (CMPD1) sur le chromosome humain 17 en q24.3-25.1 [42]. Simultanément, deux équipes, par l'analyse de nouvelles translocations, mais également par le clonage du gène $S O X 9$ murin exprimé dans les os et localisé dans une région de synténie, proposèrent $S O X 9$ comme gène candidat non seulement pour CMPDI mais également pour SRA1 (m/s $n^{\circ} 2$, vol. 11, p. 300) [43, 44]. L'existence de patients sans réarrangement chromosomique apparent a ensuite conduit ces deux équipes à rechercher d'éventuelles mutations dans le gène $S O X 9$. Ainsi furent décrites des mutations du gène, mutations hétérozygotes qui, si elles démontrent l'implication de SOXO dans le syndrome campomélique et la détermination du sexe, allongent la liste des maladies génétiques résultant de la perte d'un seul allèle (notion d'haplo-insuffisance). Si l'expression de $S O X 9$ s'effectue bien dans les structures osseuses et testiculaires, un transcrit est également présent dans d'autres tissus fœetaux et adultes, suggérant un rôle possible plus général dans les cellules de soutien. Aucune corrélation entre le type de mutation observée et le phénotype résultant, à savoir campomélisme accompagné ou non d'une réversion sexuelle, n'a pu être établie à ce jour [45]. La présence de points de translocation à plus de $130 \mathrm{~kb}$ en 5 ' de $S O X 9$ chez certains patients soulève aujourd'hui quelques interrogations sur la base moléculaire pouvant rendre compte dans ces cas du campomélisme avec réversion sexuelle: effet de position, éléments de régulations en cis très éloignés qui se trouvent détruits, etc. [46]. Quoi qu'il en soit, après la réversion du sexe et $S R Y$, la preuve est apportée de l'implication d'un deuxième gène SOX dans une anomalie du développement.

\section{Conclusion}

En conclusion, le rôle des gènes SOX dans la régulation de divers proces- 
RÉFÉRENCES

38. Stevanovic $M$, Lovell-Badge $R$, Collignon J, Goodfellow PN. SOX3 is an X-linked gene related to SRY. Hum Mol Genet 1993; 2: 2013-8.

39. Stevanovic $M$, Zuffardi $O$, Collignon $J$ Lovell-Badge $R$, Goodfellow $P$. The cDNA sequence and chromosomal location of the human SOX2 gene. Mammalian Genome $1994 ; 5: 640-2$.

40. Kamachi Y, Sockanathan S, Liu Q, Breitman $\mathrm{M}$, Lovell-Badge $\mathrm{R}$, Kondoh $\mathrm{H}$. Involvement of SOX proteins in lens-specific activation of crystallin genes. $E M B O J 1995 ; 14$ : 3510-9.

41. Yuan H, Corbi N, Basilico C, Dailey L Developmental-specific activity of the $F G F-4$ enhancer requires the synergistic action of SOX2 and Oct-3. Genes Dev 1995; $9: 2635$ 45.

42. Tommerup N, Schempp W, Meinecke P, Pederson S, Bolund L, Brandt C, Goodpasture R, Guldberg P, Held KR, Reinwein H, Saugstad OD, Scherer G, Skejldal O, Toder $R$, Westvik J, Van der Hagen CB, Wolf $U$. Assignment of an autosomal sex reversal locus (SRA1) and campomelic dysplasia (CMPD1) to $17 \mathrm{q} 24.3-\mathrm{q} 25.1$. Nature Genet $1993 ; 4: 170-4$.

43. Foster JW, Dominguez-Steglich MA, Guioli S, Kwok C, Weller PA, Stevanovic M, Weissenbach J, Mansour S, Young ID, Goodfellow PN, Brook JD, Schafer AJ. Campomelic dysplasia and autosomal sex reversal caused by mutations in an $S R Y$-related gene. Nature 1994 ; 372 : 525-30.

44. Wagner $\mathrm{T}$, Wirth J, Meyer J, Zabel B, Held M, Zimmer J, Pasantes J, Bricarelli FD Keutel J, Hustert E, Wolf U, Tommerup N, Schempp W, Scherer G. Autosomal sex reversal and campomelic dysplasia are caused by mutations in and around the $S R Y$ related gene SOX9. Cell 1994; 79: 1111-20.

45. Kwok C, Weller PA, Guioli S, Foster JW Mansour S, Zuffardi O, Punnett $\mathrm{HH}$ Dominguez-Steglich MA, Brook JD, Young ID, Goodfellow PN, Schafer AJ. Mutations in SOX9, the gene responsible for campomelic dysplasia and autosomal sex reversal. A m J Hum Genet 1995 ; 57 : 1028-36.

46. Wirth J, Wagner T, Meyer J, Pfeiffer RA, Tietze HU, Schempp W, Scherer G. Translocation breakpoints in three patients with campomelic dysplasia and autosomal sex reversal map more than $130 \mathrm{~kb}$ from $S O X 9$. Hum Genet 1996; 97 : 186-93.

47. Lecointre G, Philippe H, Van Le HL, Le Guyader H. How many nucleotides are required to resolve a phylogenetic problem? The use of a new statistical method applicable to available sequences. Mol Phylo genet Evol 1994, 3 : 292-309.

48. Philippe $\mathrm{H}$, Germot A, Le Guyader $\mathrm{H}$, Adoutte A. Que savons-nous de l'histoire évolutive des eucaryotes? 1. L'arbre universel du vivant et les difficultés de la reconstruction phylogénétique. médecine/sciences $1995 ; 11$ : I-XIII. sus de développement apparaît clairement établi aujourd'hui et cela dans diverses espèces. Les produits de ces gènes semblent capables d'intervenir dans la détermination de grands types cellulaires à partir de cellules embryonnaires. Tous semblent procéder par un mécanisme commun, permettant l'établissement d'un complexe multiprotéique (bien que ce point reste formellement à confirmer) contrôlant la transcription de tel ou tel gène cible. A ce jour, une structure classique de facteur de transcription, associant domaine de liaison à l'ADN et motif(s) de transrégulation potentiels a été retrouvée pour tous, excepté pour les SRY de mammifères non rongeurs. Gageons que, dans les mois à venir, de nouvelles données ayant trait à la découverte de nouveaux gènes $S O X$, à l'étude de leur implication dans telle ou telle voie de détermination/différenciation cellulaire, aux bases moléculaires de leur mode d'action, à la nature de leurs partenaires protéiques et de leurs cibles génétiques, ou encore à leur propre régulation seront publiées. Autant de données qui seront peutêtre particulièrement utiles à la compréhension du mode d'action de la protéine SRY au cours de la détermination du sexe, un domaine où peu de progrès notables ont été enregistrés depuis le clonage de $S R Y$. Ces gènes $S O X$ sont donc de nouveaux éléments à ajouter à la désormais longue liste des facteurs de transcrip- tion intervenant dans l'embryogenèse précoce, mais également des facteurs susceptibles de modifier localement l'organisation chromatinienne

\section{Summary}

Sex and SOX

The identification of the mammal testis determining factor SRY has led to the description of a new transcription factor family, the SOX factor family. These factors, highly conserved across evolution, were originally identified as genes carrying an HMG box closely related to that of SRY. Many SOX genes described so far have been shown to be expressed in embryonic tissues and are very likely involved in regulation of diverse developmental processes. If these SOX factors use common modes of action (binding to AT-rich DNA sequences, DNA bending, binding in the DNA minor groove), little is known about downstream targets of SOX proteins and hence about their functions. Nevertheless some SOX genes appeared already involved in developmental abnormalities such as sex reversal or campomelic dysplasia.

\section{TIRÉS À PART}

\section{P. Berta.}

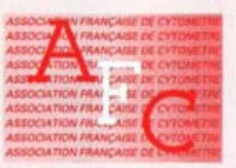

\section{ASSOCIATION FRANCCAISE DE CYTOMÉTRIE}

$3^{\text {e }}$ Congrès Annuel Cytométrie Rouen 96 16-18 octobre 1996 Rouen La Halle aux Toiles

Le troisième congrès annuel de l'AFC, Cytométrie Rouen 96, se déroulera du 16 au 18 octobre 1996 à Rouen, dans le cadre de la Halle aux Toiles, au centre historique de la ville proche de la Cathédrale. Ce congrès s'adresse à tous les membres de l'association et à toutes personnes concernées par la cytométrie dans ses aspects fondamentaux et appliqués, quelle qu'en soit l'approche technique (flux, image ou confocal).

Les sessions organisées autour des thèmes scientifiques comprendront un exposé introductif présenté par un invité spécialiste du domaine et des communications sélectionnées parmi les résumés soumis par les participants.

Les Thèmes scientifiques seront les suivants :

Avancées technologiques, traitement informatique des données, reconstructions tridimensionnelle, cellule vivante, immunologie et hématologie fondamentales, immunologie et hématologie cliniques, ADN - oncologie clinique, autres applications cliniques, prolifération, apoptose, hybridation in situ, végétaux - micro-organismes, standardisation - assurance qualite.

Des manifestations satellites auront lieu le mardi 15 octobre après-midi, et les thèmes suivants ont été retenus : Quantification en image - Numération et quantification en flux - Logiciels du cycle. Inscriptions : VISA Congrès, 624, rue des Grèzes, 34070 Montpellier, France. Tél. (33).45.56.77, Fax : (33).67.45.57.97 e.mail : Visa@imaginet.fr

Secrétariat scientifique : O.Lees, Immunologie, CHU Charles-Nicolle, 1, rue de Germont, 76031 Rouen Cedex - France - Tél. : 35.08.80.71 - Fax : 35.08.81.86 